\title{
A THEOREM CONCERNING LOCALLY PERIPHERALLY SEPARABLE SPACES*
}

\author{
BY F. B. JONES
}

Alexandroff has shown that a connected metric space is completely separable provided it is locally completely separable. $\dagger$ It is the purpose of this paper to establish a similar theorem for connected, locally connected metric spaces.

Definition. A space is locally peripherally separable provided that, if $P$ is a point of a region $R$, there exists in $R$ a domain $D$ containing $P$ such that the boundary of $D$ is separable.

THEOREM. A connected, locally connected, locally peripherally separable metric space is completely separable.

Proof. Suppose that $n$ is a fixed positive integer. Let $G$ denote the collection of all domains (to be called regions) of diameter $1 / n$ or less which are peripherally separable. Since space is locally peripherally separable, it is evident that $G$ covers space. It will now be shown that $G$ contains a countable subcollection covering space. For each point $X$ of space let $n_{x}$ denote the largest integer such that no region of $G$ contains a circular domain with center at $X$ and radius greater than or equal to $1 / n_{x}$. Let $D_{1}$ denote some region of $G$. For each integer $i$ let $M_{1 i}$ denote the set of all points $X$ of the boundary $\beta_{1}$ of $D_{1}$ such that $n_{x}=i$. Since space is metric and $\beta_{1}$ is separable , $\beta_{1}$ is completely separable, and there exists in $M_{1 i}$ a countable point set $N_{1 i}$ which is everywhere dense in $M_{1 i}$. Now for each point $X$ of $N_{1 i}$ let $R_{x}$ denote a region of $G$ containing a circular domain with center at $X$ and radius $1 /(i+1)$. The sum of these regions $R_{x}$ forms a domain $Q_{1 i}$ covering $M_{1 i}$, and $\sum_{i=1}^{\infty} Q_{1 i}$ is a domain covering $\beta_{1}$.

Let $D_{2}=D_{1}+\sum_{i=1}^{\infty} Q_{1 i}$. Then $D_{2}$ contains $D_{1}+\beta_{1}$. Since space is locally connected, every point of the boundary $\beta_{2}$ of $D_{2}$ either belongs to the boundary of some region $R_{x}$ or is a limit point of

* Presented to the Society, June 20, 1934.

† Paul Alexandroff, Über die Metrization der im kleinen kompakten topologischen Räume, Mathematische Annalen, vol. 92 (1924), pp. 294-301. 
the sum of the boundaries of these regions $R_{x} *$ Furthermore, there are only countably many regions $R_{x}$. Hence, $\beta_{2}$ is separable. It is also to be noted that $D_{2}$ is the sum of countably many regions of $G$.

This process may be continued. Thus there exist a sequence $D_{1}, D_{2}, D_{3}, \cdots$ of domains and for each integer $m$ a sequence $N_{m 1}, N_{m 2}, N_{m 3}, \cdots$ of point sets, so that if, for each integer $m$, $\beta_{m}$ denotes the boundary of $D_{m}$, and, for each pair of integers $m$ and $i, M_{m i}$ denotes the set of all points $X$ of $\beta_{m}$ such that $n_{x}=i$, then

(0) $D_{m}$ is the sum of countably many regions of $G$,

(1) $D_{m}$ contains $D_{m-1}+\beta_{m-1}$,

(2) $\beta_{m}=\sum_{i=1}^{\infty} M_{m i}$,

(3) $N_{m i}$ is a countable subset of $M_{m i}$ which is everywhere dense in $M_{m i}$,

(4) if $X$ is a point of $N_{m i}, D_{m+1}$ contains a circular domain with center at $X$ and radius $1 /(i+1)$, and

(5) if $R$ is a region of $G$ containing a point $X$ of $N_{m_{\imath}}$, no circular domain $V_{x}$ lying in $R$ with center at $X$ has a radius greater than or equal to $1 / i$.

Suppose now that there is some point of space not belonging to any $D_{m}$. Then, since space is connected, there exists a point $O$ on the boundary of $\sum D_{m}$. Hence, it follows from (1), (2), and (3) that $O$ is a sequential limit point of a sequence of points $X_{1}, X_{2}, X_{3}, \cdots$, where for each $m, X_{m}$ belongs to $N_{m i}$ for some $i$. Now, let $R$ denote a region of $G$ containing a circular domain $V_{0}$ with center at $O$ and radius $r$. For each point $X_{m}$ of the sequence $X_{1}, X_{2}, X_{3}, \cdots$ let $V_{X_{m}}$ denote a circular domain with center at $X_{m}$ and radius $r / 2$. There exists a number $k$ such that if $m>k$, then $V_{X_{m}}$ lies in $R$. However, since $O$ is not covered by $\sum D_{m}$ and $N_{m i}$ contains $X_{m}$, it follows from (4) that $i$ increases with $m$. Hence, for some point $X_{m},(m>k), r / 2$ is greater than $1 / i$. This contradicts $(5)$; so $\sum D_{m}$ covers space. By (0), $D_{m}$ is the sum of countably many regions of $G$, and therefore $\sum D_{m}$ is the sum of the regions of a countable subcollection $G^{\prime}$ of $G$. Hence $G^{\prime}$ covers space.

Now, for each integer $n$, let $G_{n}$ denote the collection of periph-

* R. L. Moore, Foundations of Point Set Theory, Colloquium Publications of this Society, vol. 13, 1932, Theorem 2(b), Chapter II, p. 91. 
erally separable domains of diameter $1 / n$ or less. By the above argument each $G_{n}$ contains a countable subcollection $G_{n}^{\prime}$ covering space. Hence, space is completely separable and the theorem is established.

The University of Texas

\section{A REDUCED SET OF POSTULATES FOR ABSTRACT HILBERT SPACE*}

\section{BY A. E. TAYLOR}

1. Introduction. An abstract Hilbert space is a normed linear space, or vector space, of infinite dimensionality, with a norm based on a Hermitian inner product, defined for all pairs of elements in the space. The space is, moreover, separable and complete according to this norm. The usual postulate system for Hilbert space, which was first stated abstractly by J. von Neumann, consists of five groups of postulates, or nineteen in all. $\dagger$

The purpose of the present paper is to demonstrate the redundancy $\ddagger$ of a number of the postulates, and to present a system of eleven independent postulates for a normed linear space with a Hermitian inner product. The adjunction of three more postulates, each of which is independent of the first eleven and the remaining two, then gives us a system which is equivalent to that of von Neumann, that is, it defines an abstract Hilbert space, and it is categorical.

A special feature of this postulate system is that the abstract relation called equality, and denoted, as usual, by the symbol $=$, enters on an equal footing with the operations defined in the space. $\S$ Three of the eleven postulates are concerned with this

* Presented to the Society, December 1, 1934.

$\dagger$ J. von Neumann, Mathematische Grundlagen der Quantenmechanik, 1932. pp. 19-24; M. H. Stone, Linear Transformations in Hilbert Space, Colloquium Publications of this Society, vol. 15, pp. 2-4.

$\ddagger$ Some of these redundancies were noted simultaneously by a fellowstudent, Mr. Ivar Highberg, and myself.

$\S$ The postulational treatment of equality in vector spaces was suggested by A. D. Michal in a critique of postulate systems. See this Bulletin, vol. 39 (1933), Abstract No. 339. 